\title{
Journal of Real-Time Image Processing: fourth issue of volume 13
}

\author{
Matthias F. Carlsohn ${ }^{1} \cdot$ Nasser Kehtarnavaz $^{2}$
}

Published online: 28 October 2017

(C) Springer-Verlag GmbH Germany 2017

This is the fourth and final issue of volume 13. In 2016, two volumes consisting of eight issues were published in order to reduce the backlog of online first published articles waiting to appear in print. For the same reason, it is planned to offer two volumes of JRTIP next year, that is volume 14 and volume 15 , each volume consisting of four issues for a total of eight issues in 2018. The additional volume or four issues will increase the total number of print pages to 1600 in 2018 which will greatly alleviate the backlog of accepted papers that have already appeared online and are waiting to appear in print. This step is being taken as part of our efforts to steadily shorten the time between the online and print versions of accepted articles.

As normally done in the last few years, the editorial board of JRTIP will be meeting at the SPIE Conference on Real-time Image and Video Processing as part of the SPIE umbrella symposium on Defense and Commercial Sensing to be held in April 2018 in Orlando, FL, USA. The call for papers for the conference and later the tentative program of the conference can be found at this link: http://spie.org/ SIC/conferencedetails/real-time-image-video-processing.

This call for paper is also included in the backmatter of this issue. We will report on the outcome of this meeting in an editorial appearing after the meeting.

Before providing a brief outline of the papers in this issue, we are pleased to mention that the newly released ISI

Matthias F. Carlsohn

matthias.carlsohn@t-online.de

Nasser Kehtarnavaz

kehtar@utdallas.edu

1 Engineering and Consultancy for Computer Vision and Image Communication, Bremen, Germany

2 University of Texas at Dallas, Richardson, TX, USA impact factor (IF) for JRTIP has gone back up to 2.010. The increase in the number of articles to appear in print in 2018 due to the additional volume is anticipated to have a positive impact on the impact factor.

In order to further improve the turnaround time of the review process and the quality of the reviews, we would like to remind readers of the Reviewer Reward Programme whose details had been mentioned in our previous editorials and are described at this link: http://www.springer. com/computer/image+ processing/journal/11554/PSE?de tailsPage $=$ press.

Currently, four special issues are being conducted by several guest editor teams to highlight the hot spot topics in real-time image processing. The calls for papers for these special issues can be found at this link: http://www. springer.com/computer/image+processing/journal/11554/ PSE?detailsPage $=$ societies and in the backmatter of this issue as well. Furthermore, we would like to encourage colleagues and researchers to submit proposals for special issues. Such issues provide a collection of focused articles on contemporary subjects of interest as related to the realtime aspects of image and video processing such as computational complexity reduction compared to existing solutions, real-time hardware implementation on various processors or platforms, actual real-time processing rates, and real-time software optimization.

This final issue of volume 13 is a regular issue which comprises 10 original research articles addressing different image processing topics related to the real-time theme of JRTIP.

The first article by Elloumi et al. is on "Real-time camera orientation estimation based on vanishing point tracking under Manhattan World assumption" and addresses a real-time pipeline for estimating the camera orientation based on vanishing points for indoor navigation 
assistance on smartphones. The discussed method is based on finding reliable triplets of orthogonal vanishing points combined with a particular sampling strategy and tracking along a video sequence by extracting the three most pertinent orthogonal directions under real-time constraints. The results of experiments on images captured by a smartphone are provided for this navigation application.

The second paper on "Real-time, parallel segmentation of high-resolution images on multi-core platforms" by Fitzgerald et al. presents an optimized serial implementation of leap segmentation, which achieves frame rates exceeding more than $80 \mathrm{fps}$ on $640 \times 360$ images and more than $20 \mathrm{fps}$ on high-resolution $(1280 \times 720)$ images. Additional speed-up could be achieved on a multi-core, mobile processing system with four threads, leading to frame rates of over $114 \mathrm{fps}$ on $640 \times 360$ images and more than $31 \mathrm{fps}$ on $1280 \times 720$ images, thus exceeding realtime demands of video processing.

The third paper by $\mathrm{He}$ et al. on "A combinational algorithm for connected-component labeling and Euler number computing" combines two processing tasks that are usually executed independently by different algorithms for labeling connected components in a binary image and computing the Euler number of the image. This approach simultaneously exploits the same neighborhood information obtained from its adjacent pixels in one single scan where intermediate results of current pixel information always provides input for subsequent pixels. The experiments conducted demonstrate the simplicity and efficiency of the developed method compared to conventional methods.

The fourth article on "GPU-accelerated denoising of 3D magnetic resonance images" by Howison et al. is dedicated to finding the appropriate parameters for best denoising results in 3D MR images using bilateral filtering, anisotropic diffusion, and non-local means. It also discusses how to tune this process on GPUs by using mean squared error (MSE) and mean structural similarity (MSSIM) as quality criteria and to tune optimal performance on contemporary GPUs by empirically autotuning to optimal memory tiling on GPUs. The results reported suggest tuning is as essential step in achieving real-time performance with corresponding implications for the real-time application of denoising to MR images in clinical settings that require fast turnaround times.

The fifth paper entitled "Real-time field sports scene classification using colour and frequency space decompositions" by Kapela et al. is related to scene classification in field sports video. It presents a novel approach to recognize a scene presented in an image by proposing to take into account only the most important areas of similar colour as a real-time simplification approach while achieving similar accuracy with well-known image indexing techniques like
SIFT or HoGs. The discussed approach originates from using very well-known Fourier transform and making it a good candidate for real-time video indexing systems.

The sixth article with the title "Adequation and hardware implementation of the color structure descriptor for real-time temporal video segmentation" by Ben Abdelali et al. is focused on the study of the MPEG-7 color structure descriptor (CSD) proposing a new hardware architecture. This new hardware architecture of the CSD is proposed with the aim of optimizing its implementation in terms of hardware resources and execution time. It is applied for different quantization levels in the HMMD color space and for different grey levels, with and without a frame skip where a low number of quantization levels and frame skipping significantly reduces the complexity, achieving better computing performance while preserving a satisfactory level of accuracy in terms of shot boundary detection rate. This is essential for implementation on resource-constrained hardware platforms and multiprocessing applications. The performance of the proposed architecture was evaluated for different quantization levels to show the effect on occupied hardware resources and execution time. A comparative study conducted demonstrates the effectiveness of the proposed architecture, which can operate video in real-time and without restriction on image size. Finally, a system on chip (SOC) is designed for real-time video summarization based on the CSD integrating the CSD module on a platform based on a Xilinx Virtex5 FPGA. A complete demonstration, including CSD extraction, shot boundary detection and key-frames visualization, is also realized.

The seventh paper is on "FestGPU: a framework for fast robust estimation on GPU" where Roters et al. are presenting a framework for Fast robust ESTimation on GPU, which achieves a speedup by a factor of 135 compared to a single core $\mathrm{CPU}$ with a $\mathrm{C}++$ and a Matlab interface. RANSAC is examined noting that it is a widely used algorithm for robust estimation achieving a high degree of accuracy even with a significant amount of outliers. The framework is made publicly available on the authors' website for the research community.

The eighth paper on "Real-time fingerprint image enhancement with a two-stage algorithm and block-local normalization" by Kočevar et al. presents a block-local normalization algorithm and a technique for speeding up a two-stage algorithm for low-quality fingerprint image enhancement with image learning, enhancing first a fingerprint image in the spatial domain and subsequently in the frequency domain. The normalization technique includes an algorithm with block-local normalization using different block sizes. The experimental results obtained on a public database $\mathrm{FVC2004} \mathrm{show} \mathrm{that} \mathrm{the} \mathrm{normalization}$ technique speeds up and improves a state-of-the-art two- 
stage algorithm and provides better results in comparison to global and local normalization which affects fingerprint image enhancement positively improving the entire efficiency of the automated fingerprint identification system.

In the ninth paper Castillo Atoche et al. are presenting "An efficient systolic array grid-based structure of the robust Bayesian regularization technique for real-time enhanced imaging in uncertain remote sensing environment" in which a hardware implementation of the efficient robust Bayesian regularization architecture for the realtime enhancement of large-scale remote sensing (RS) imaging is covered. The efficiency of the proposed architecture originates from the aggregation of parallel computing and systolic array design techniques in a novel grid connected-based accelerator. This high-speed accelerator is integrated within an embedded processor via the HW/SW co-design paradigm. The presented approach is used for solving RS image enhancement/reconstruction of the illconditioned inverse spatial spectrum pattern estimation problems via an interesting low-cost high-performance embedded computing solution. Finally, the results obtained demonstrate the drastic reduction in the computational load for real-world large-scale geospatial images.

The tenth and final paper of this issue by Yang et.al. entitled "Fast intra encoding decisions for high efficiency video coding standard" deals with HEVC as the nextgeneration standard developed by the Joint Collaborative Team in Video Coding (JCT-VC) aiming to achieve 50\% bit rate saving with similar objective quality compared to H.264/AVC. A flexible quadtree coding structure characterized by coding units (CUs), prediction units (PUs) and transform unit (TUs) is adopted by HEVC introducing a high computational complexity depending on the decision modes of optimal CU, PU and TU. To reduce the intra encoding complexity, a solution is proposed that includes fast CU skip decision, fast CU early termination, fast PU mode decision and fast TU size decision. The experimental results demonstrate that the proposed solution achieves on average $65 \%$ total encoding time reduction with less than $1.3 \%$ on average BD rate increase. 